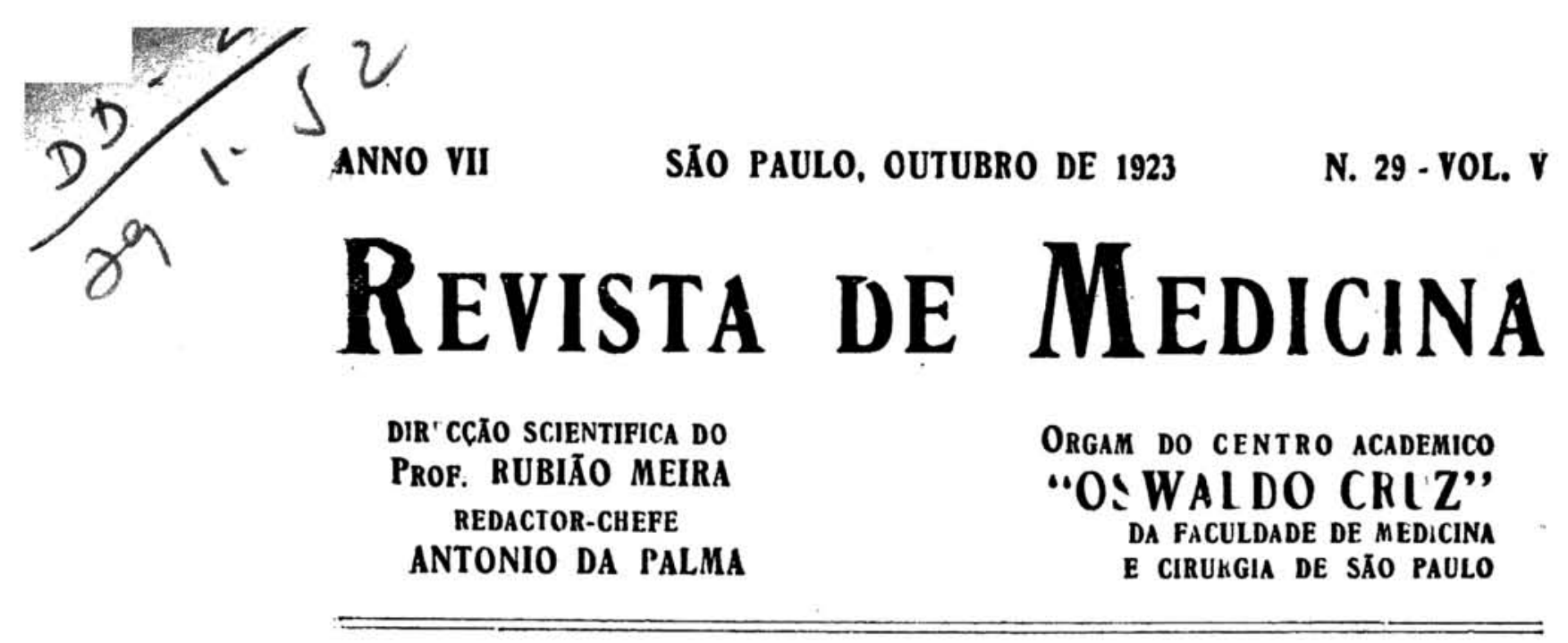

\title{
A MISSÃO INTELLECTUAL FRANCEZA
}

o governo francez e o governo brasileiro, conjuntamente, deliberaram crear nas capitaes dos dois paizes - Paris e Rio de Janeiro o "Instituto Franco-brasileiro de Alta Cultura".

$E^{\prime} a$ idèa tão defendida pelo illustre medico $e$ puólicista francez, Dr. Jorge Dumas, $\epsilon$ applaudida por tantos francezes $e$ brasileiros, que, anıparada agora pelos dois governos, transforma-se $\mathrm{cm}$ realidade con!pleta.

o livro, toda a gente o sabe, é o melhor meio de propaganda de que póde dispôr modernamente um povo para a conquista da intelligencia e do coração de outro povo...

¿Que novidade haveria se accrescentassemos que, depois do livro, o professor é outra grande força nesta ordem de trabalho?

Pois bem: si considerarmos, a seguir, este facto patente: - a França continúa a representar na vida do pensamento universal o mesino papel que the vem cabendo, ha seculos, de alto e vigoroso expoente de um grupo de povos - havemos de comprehender em como, para esse paiz ' uma valiosa obra, esta, de firmar em bases ainda mais estaveis a iá inequivoca admiração e enthusiasmo do nosso paiz ipelas gentes qu.e hubitam entre os Pyrinéos e o Rheno, o Mar da Mancha $e$ os Alpes.

Nos dias de após-a-guerra, que são os que estamos vivendo, e em que e preciso falar commercialmente para ter-se a certeza de ser bem cntendido, é um intelligente passo o que ora dá o governo francez, pois assenhoreiam-se, por elle, ainda mais, as letras francezas de um mercado ja intciramente seu - o mercado de livros do Brasil.

His um lado da questão, examinado em uma das suas modalidades. outros lados ha, $e$ nelles outras vantagens existem a que poderiamos alludir.

Mas deixemol-o...

水

${ }_{6}$ O Brasil, estará de cumprimentos pela parte de proveitos que the aevem caber na creação do Instituto Franco-brasileiro de Alta Cultura?

- Certamente, cremos nós, e aina mais do que a França...

Para não falar doutras, parece-nos que duas consequencias, ambas de muito alcance, terá este instituto para a mentalidade nacional das tiscolas superiores. 
Antes de mais, o Instituto Franco-brasileiro de Alta Cultura vem dar aos nossos meios universitarios caracter mais accentuadamente universitario que lhes falta.

Os cursos syntheticos e internacionaes, como os do Collegio de França, onde se faz a revisão geral dos estudos $e$ os conhecimentos se orientam e firmam melhor, cursos faceis de accesso e simples pelo regimento condiçōes optimas para a vulgarisação do saber - têm, a partir deste momento, entre nós, para diffusāo da cultura franceza, um auctorisado representante no Instituto Franco-brasileiro de Alta Cultura. (Entende-se immediatamente que, "mutatis mutandis", o mesmo se diz dos assumptos brasileiros na França).

$E$ o Brasil, $e$ a mocidade brasileira das Escolas Superiores estão, pois, de parabens.

Nesta primeira vantagem contêm-se uma outra não menos apreciavel.

Da mais intima approximação que desta fórma - o Instituto Francobrasileiro - se crêa entre as duas nacionalidades, resulta, afinal, $o$. abreviar-se a differença que existe no tempo entre o nivel geral da instrucção do nosso paiz e o da França. Esta se encontra entre os mais activos centros de progresso mental da Europa (Europa que, digase de passagem, neste particular ainda não perdeu a deanteira em todo o mundo).

Nós, descrevendo cyclos de evolução semelhantes aos outros povos (o homem $=0$ homem) mantemos em relação a uns, (no caso, a França uma certa distancia, para traz, - o que sendo veridico, natural e logico, de modo nenhum ros deve melindrar...

Ora, deante da innegavel influencia que todos sentimos da França sobre o Brasil, näo teri este a ganhar, desde que se the proporcione condiçöes de mais brevemente e melhor realisar os estados evolutivos nor que ha de necessarianiente passar - n'uma época em que o cosmopolitismo sempre crescente obriga os paizes mais novos a adoptar immediatamente todos os progressos dos mais velhos?...

P.

\section{ANNUNCIEM NA "REVISTA DE MEDICINA"}

Mediante pedido enviamos tabellas de preços e prestamos promptamente quaesquer outras informações. 


\section{SCIENTISTAS FRANCEZES VISITAM SÃO PAULO}

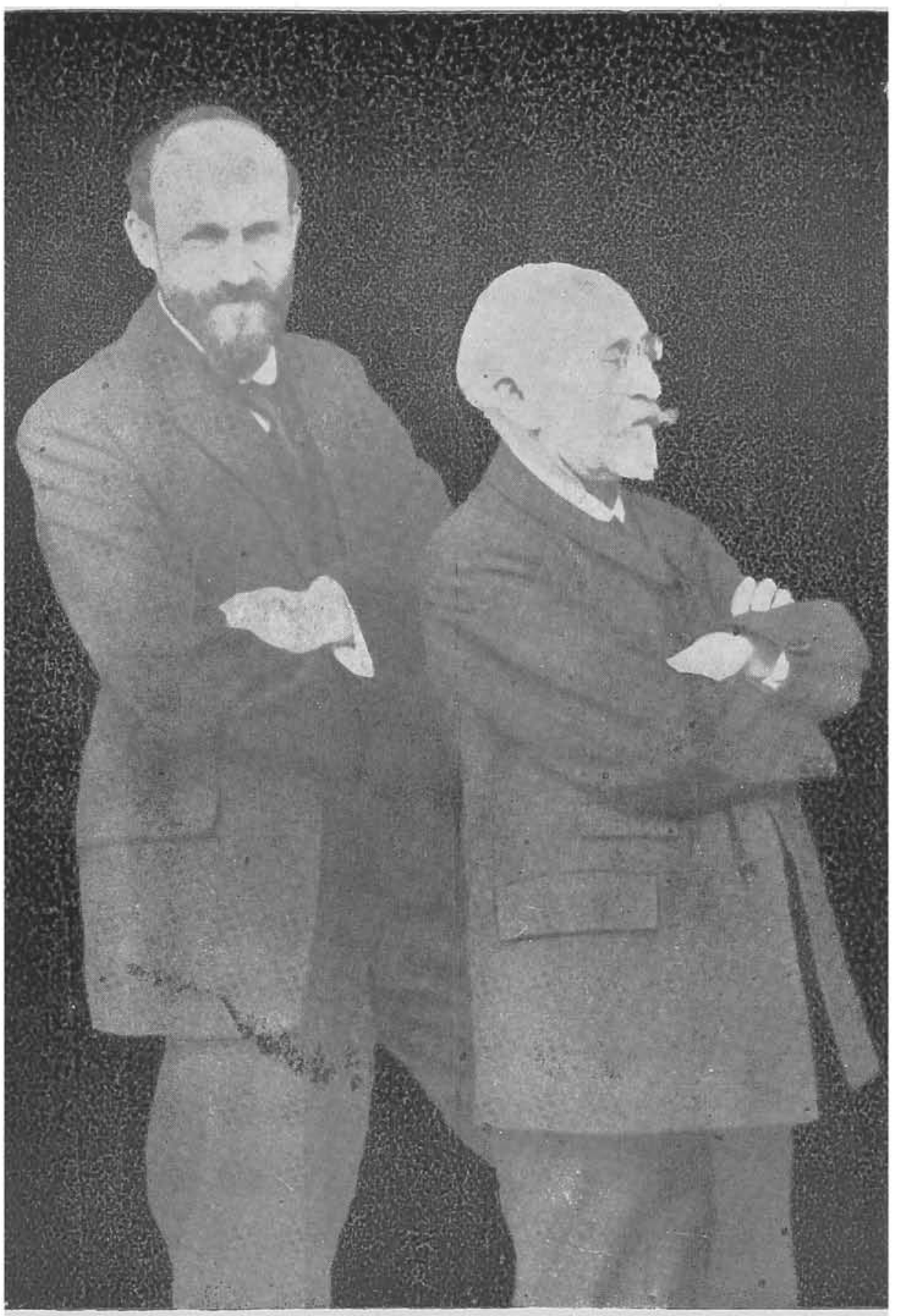

Os eminentes professores Henrique Piéron (á esquerda) e Eugenio Gley (á direita), posando para o photographo da "REVISTA DE MEDICINA". 



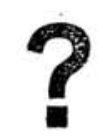

A respeito do professur Eugenio Gley, o conhecido physiologista cujo tratado sobre a materia é, seguramente, para os estudantes de Medicina do Brasil, um verdadeiro "ivro de horas" - uma aas curiosidades mais humanas dos mocos da Faculdade (e mesmo fóra della) foi certamente, "ver o homem", e, depois de o ver, ouvil-o.

$A$ assistencia numerosissima que accorreu ás duas conferencias que o notavel scientista fez em São Paulo bem o mostraram.

ora, é justamente indo ao encontro desta curiosidade, de certo legitima, que resolvemos completar a mão e aqui apresentamos aos nossos leitores uma pagina escripta especialmente para $a$ "REVISTA DE MEDICINA". Por ella $o$ eminente professor responde a uma outra curiosidade, a nossa, quando the perguntamos algures quaes seriam, na sua opiniāo, os proveitos certos para os dois paizes, a França e o Brasil, da creação, entre nós, do "Instituto Francobrasileiro de Alta Cultura", cujos cursos elle foi um dos scientistas francezes encarregados de inaugurar

\begin{abstract}
*
Le repprochement des peuples est le but lointain auquel tend l'humanité. Ce sont les relations intéllectuelles qui réaiisent le mieux cette grande cause des activités internationales.

On ne bâile que lorqu'on ne sait pas, a dit Spinoza.

Entre peuples de même race ce rapprochement est d'abord plus facile. L'Institut Franco-brésilien aura pour résulta essentiel le resserrement des liens qui existent déjà entre les deux peuples de la même origine latine et la commune culture et le même idéal.

Les français présenteront au Brésil des exemples de leur manière rationelle de considérer toutes les questions, de les étudier et de les exposer suivant l'ordre logique; des exemples aussi de leur esprit de mesure, de leur sens esthétique; des exemples infin de leur enthousiasme persistant pour les recherches désintéressés dans le domaine de la science comme dans celui de l'indution ou dans les speculations philosophiques. Ft les Brésiliens qui viendront à Paris représenter à l'Institut Franco-brasilien leur grande patrie, auront pour premier office de faire mieux connaître à la France l'historie de leur pays et de son développement matériel, les problèmes économiques qui se' posent sur une terre aussi étendue, la façon dont ses immenses ressources sont exploitées, les nombreaux et importants problèmes scientifíques en géologie, en botanique, en biologie, en agronomie, en pathologie humaine et comparée qui se présentent devant l'esprit de quiconque pénètre au Brésil et commence á le comprendre et á l'aimer et qui. doivent se présenter à l'esprit de ses classes dirigeantes.
\end{abstract}




\section{$?$}

Ao eminente psychologo experimentalista, collega do grande pesquizador Engenio Gley no magisterio no Collegio de França, Henrique Piéron, offerecemos nós, tambem, uma pequena pergunta que restiondida. deveria dar. como o dá agora tıma pagina inedita para os leitores da "REVISTA DE MEDICINA"

$A$ pergunta foi esta: Après cette dernière grande guerre qui vient de finir, quelle est à votre avis la direction que suivra

l'espirit humain dans domaine des sciences expérimentales?

$E^{\prime}$ a resposta com que entendeu honrar-nos o Dr. Henrique Piéron que abaixo offerecemos aos nossos leitores.

La guerre est terminée depuis cinq années, et bien que ses séquelles doivent durer sans doute longtemps encore, ou peut juger déjà, d'après l'orientation actuelle des recherches, les effets possibles de la grande conflagration mondiale.

Or, si momentanement les difficultés économiques et les pertes de vie humaine ont diminué le nombre des rechercheurs, et relativement paralysé les simples travaux d'applicaiton, dont le rendement immédiat apparait plus certain, le grand ouvre scientifique a reprit partout, et le culte idéaliste de la verité renait.

Nous pouvons avoir pleine confiance dans la marche continue du progrès des connaissances humaines; il y aura eu quelques années de relentisement qui se marqueront dans l'histoire comme les maladies individuelles se traduisent par un retard longtemps observable dans la croissance des phanères; ce n'a été là qu'une. perturbation passagère, et, lorque l'ouragan est passé, les plantes se redressent et poussent, dressant leur cime vers le ciel!

Henri Piéron.

\section{ASSIGNEM A "REVISTA DE MEDICINA" \\ BRASIL (12 numeros) . . . . . . . $18 \$ 000$ \\ ESTRANGEIRO . . . $\quad 36 \$ 000$

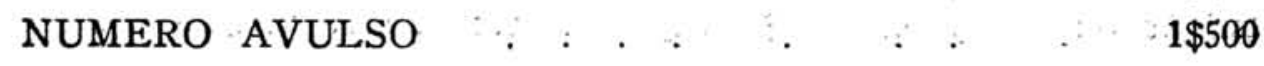

